\title{
Pratiques
}

Linguistique, littérature, didactique

\section{L'enseignement de la littérature en dialogue avec les arts : des discours institutionnels à harmoniser}

Teaching litterature along with arts: institutional discourses (on the issue) need to be harmonized

\section{Sylviane Ahr}

\section{OpenEdition}

\section{Journals}

Édition électronique

URL : http://journals.openedition.org/pratiques/3568

DOI : 10.4000/pratiques.3568

ISSN : 2425-2042

Éditeur

Centre de recherche sur les médiations (CREM)

Référence électronique

Sylviane Ahr, « L'enseignement de la littérature en dialogue avec les arts : des discours institutionnels à harmoniser », Pratiques [En ligne], 175-176 | 2017, mis en ligne le 22 décembre 2017, consulté le 01 mai 2019. URL : http://journals.openedition.org/pratiques/3568 ; DOI : 10.4000/pratiques.3568

Ce document a été généré automatiquement le 1 mai 2019.

(c) Tous droits réservés 


\title{
L'enseignement de la littérature en dialogue avec les arts : des discours institutionnels à harmoniser
}

Teaching litterature along with arts: institutional discourses (on the issue) need to be harmonized

\author{
Sylviane Ahr
}

1 Nombreux sont les discours institutionnels, publiés ces toutes dernières années, qui tendent à promouvoir la mise en dialogue de l'enseignement de la littérature avec les arts. Ces discours concernent tout aussi bien la classe que la formation universitaire préparant au concours de recrutement des enseignants de français. En effet, depuis la session 2014, sont proposées aux candidats admissibles du Capes externe de lettres modernes $^{1}$ deux nouvelles épreuves qui, comme le souligne le président du jury des sessions 2014 et 2015, «manifestent un souci d'ouverture aux évolutions culturelles de la discipline et de ses pratiques professionnelles» (Blanckeman, 2014, p.6). Outre «la systématisation du rapport texte/image par le biais des documents iconographiques que les candidats doivent faire dialoguer avec les extraits d'œuvre étudiés lors des deux épreuves orales ", les options théâtre et cinéma de la seconde épreuve ${ }^{2}$ doivent favoriser « de fertiles échanges transdisciplinaires » (ibid., p. 6-7). De même, la rénovation du Socle commun de connaissances, de compétences et - désormais - de culture ainsi que la mise en application de nouveaux programmes, en particulier au collège, à la rentrée 2016 tendent à «situer l'enseignement des lettres et du français dans un champ culturel multipolaire » (ibid.), perspective selon laquelle sont à concevoir dorénavant les épreuves d'admission du Capes. L'institution semble donc, au niveau du concours de recrutement des professeurs comme dans ses prescriptions actuelles, ouvrir l'enseignement des lettres et du français aux « autres domaines artistiques » (MENESR, 2015c, p. 121). Cependant, les modalités selon lesquelles candidats au concours de recrutement et enseignants en exercice sont invités à réaliser des «rapprochements entre les œuvres, littéraires, iconographiques et cinématographiques»(ibid., p. 111) répondent-elles aux mêmes enjeux ? Dans les deux cas, qu'entend l'institution par mise en « dialogue entre les arts » 
(MENESR, 2015c, p. 237), que le (futur) professeur de français doit favoriser, aussi bien au collège qu'au lycée? Après une rapide présentation du corpus constitué, l'analyse comparée des rapports de jury des deux épreuves orales du Capes externe de lettres modernes des trois dernières sessions $\left(2014-2016^{3}\right)$ et des divers textes et documents officiels cadrant aujourd'hui l'enseignement de la littérature dans le secondaire visera à mettre au jour les convergences et les divergences perceptibles dans ces discours institutionnels, qui, d'une façon ou d'une autre, déterminent les pratiques de classe en matière d'enseignement de la littérature en lien avec les autres œuvres artistiques.

\section{Les discours institutionnels actuels}

2 Rendu obligatoire de l'école au lycée par la loi d'orientation et de programmation pour la refondation de l'école de la République du 8 juillet 2013, le parcours d'éducation culturelle et artistique (PÉAC) « a pour ambition de viser un égal accès de tous les jeunes à l'art et à la culture » (MEN, 2013a). Pour accompagner la mise en œuvre de ce parcours est édité dès 2013 un Guide qui recommande aux enseignants « de veiller à la diversité des grands domaines artistiques et culturels abordés par les élèves tout au long de leur scolarité - différentes formes du spectacle vivant, arts visuels, arts du son, arts de l'espace, arts appliqués, arts du langage » (MEN, 2013b, p. 8). Afin de faciliter la mise en œuvre du PÉAC - qui concerne l'ensemble des niveaux de la scolarité, y compris les classes des filières technologiques et professionnelles du lycée -, sont proposés aux enseignants plusieurs exemples de projets : ceux-ci accordent, en particulier au collège et au lycée, une large place à la discipline « français ». En outre, à l'initiative du Haut conseil de l'éducation artistique et culturelle est diffusée en 2016 une Charte qui pose les dix principes autour desquels doit s'organiser l'éducation à l'art et par l'art (MENESR et al., 2016).

3 En 2015, le ministère réaffirme la nécessité d'inscrire le PÉAC « dans le projet global de formation de l'élève défini par le socle commun de connaissances, de compétences et de culture et opérationnalisé par les programmes de cycle »(MENESR, 2015b). La rénovation du socle en 2015 (MENESR, 2015a) ainsi que la mise en application de nouveaux programmes, en particulier au collège, à la rentrée 2016 tendent à situer l'enseignement des lettres et du français dans un champ culturel et artistique ouvert. Les programmes de français de 2015 pour les cycles 3 et 4 précisent en effet que les lectures d'œuvres littéraires «s'organisent autour d'entrées qui appellent les mises en relation entre les textes et d'autres documents ou œuvres artistiques » (MENESR, 2015c, p. 109) : «l'une des finalités majeures de l'enseignement du français » (ibid., p. 246) est « la constitution d'une culture littéraire et artistique commune, faisant dialoguer les œuvres littéraires du patrimoine national, les productions contemporaines, les littératures francophones et les littératures de langues anciennes et de langues étrangères ou régionales, et les autres productions artistiques, notamment les images, fixes et mobiles» (ibid., p. 229). Cette orientation confirme celle déjà retenue - même si de façon moins accentuée - dans les programmes du lycée général et technologique mis en application à la rentrée 2011. Ceuxci recommandent aux professeurs de faire percevoir aux lycéens « les correspondances entre les arts et la singularité de leurs modes et de leurs formes d'expression»; de montrer, par exemple, aux élèves de seconde « comment l'esthétique réaliste concerne plusieurs formes d'expression artistique », de les rendre " sensibles aux liens qui unissent la poésie aux autres arts, à la musique et aux arts visuels » (MEN, 2010). Enfin, il n'est pas 
inutile de rappeler que cette mise en dialogue de la littérature et des arts concerne aussi l'enseignement du français dans les classes préparatoires au baccalauréat professionnel, comme le suggère cet extrait des programmes de 2009, actuellement en application ${ }^{4}$ :

En lien avec les enseignements d'histoire, de géographie, d'éducation à la citoyenneté, de langues vivantes, d'arts appliqués, d'histoire des arts, l'enseignement du français participe à l'enrichissement de la culture commune par la connaissance de mouvements et d'œuvres, par la fréquentation de productions artistiques variées, par la pratique d'activités culturelles. (MEN, 2009, p. 1)

L'un des objets d'étude de la classe de terminale professionnelle, « Au XX siècle, l'homme et son rapport au monde à travers la littérature et les autres arts », favorise explicitement le rapprochement entre les différents langages artistiques.

Ces divers textes officiels s'inscrivent bien évidemment dans la logique institutionnelle initiée en 2008 par l'introduction de l'enseignement de l'histoire des arts (et non de l'art) dans le système éducatif français. Mis en place à l'école primaire dès la rentrée 2008 puis au collège et au lycée à la rentrée 2009, cet « enseignement de culture artistique partagée [...] est porté par tous les enseignants » (MEN, 2008, p. 1) et a fortiori par ceux de français. On ne reviendra pas sur la polémique suscitée par l'entrée de cet enseignement dans les classes et relayée par la presse ${ }^{5}$, en particulier lors de l'installation, à la session 2011, d'une épreuve d'histoire des arts au diplôme national du brevet des collèges. Cependant, comme le fait remarquer alors H. de Rohan-Csermak (2010), Inspecteur général de l'éducation nationale pour l'histoire des arts, le risque demeure qu'en raison de la configuration propre aux établissements du secondaire, cet « enseignement fondé sur une approche pluridisciplinaire et transversale » ne conduise les professeurs à « utiliser les arts comme une illustration des enseignements disciplinaires». Tendance que visent à pallier les discours institutionnels récents, liés à l'introduction du PÉAC et de nouveaux programmes d'enseignement adossés à un socle rénové ainsi qu'à la mise en place des Enseignements Pratiques Interdisciplinaires (EPI) au cycle 4. Y a-t-il néanmoins consensus quant au statut à accorder aux arts dans le cadre de l'enseignement de la littérature, tel que le définissent les programmes actuels du collège (2015) et ceux du lycée général et technologique (2010) d'une part, tel que le conçoivent les jurys du concours de recrutement des professeurs certifiés de lettres modernes depuis 2014 d'autre part?

\section{Convergences et divergences dans les discours institutionnels}

6 L'analyse comparée des textes et documents officiels et des rapports de jurys conduit à interroger ce que l'institution entend par « enseigner la littérature en dialogue avec les arts ", lorsqu'il s'agit d'adresser des recommandations aux professeurs en exercice, des conseils aux candidats admissibles du Capes externe de lettres modernes. À quels enjeux en termes d'enseignement et d'apprentissage ce "dialogue entre les arts " (MENESR, 2015c, p. 237), à initier dans les classes de littérature, doit-il répondre? Selon quelles modalités doit-il, peut-il se réaliser? Les acteurs institutionnels tiennent-ils un discours commun, voire cohérent, sur la nécessaire ouverture de la discipline aux autres formes artistiques? 


\section{Quelle mise en dialogue de la littérature avec les arts ?}

7 Les programmes de français du collège (du cycle 4 mais aussi du cycle 3 pour ce qui concerne la classe de sixième) de 2015 soulignent la nécessité de " mettre en relation », de «faire dialoguer» les œuvres littéraires et les autres créations artistiques, de « solliciter les rapprochements " entre les unes et les autres, de développer les « ouvertures vers d'autres domaines artistiques»(MENESR, 2015c, p. 109, 111, 121, 229, 237). Mais ce « dialogue entre les arts » ne semble concerner - du moins dans les programmes - que les œuvres iconographiques et cinématographiques, même si la pratique culturelle du théâtre est fortement encouragée. Il est ainsi précisé :

Chaque année, dans le cadre du parcours d'éducation artistique et culturelle, une œuvre cinématographique au moins est vue et étudiée par la classe. Au cours du cycle, si l'offre culturelle le permet, les élèves assistent à au moins une représentation théâtrale. À défaut, des captations peuvent être utilisées. (ibid., p. 109)

Images fixes ou mobiles sont donc à privilégier, et on en comprend aisément la raison : leur utilisation dans les classes est facilitée par les équipements numériques dont celles-ci bénéficient généralement aujourd'hui. Les programmes du cycle 4 rappellent également :

Les images fixes ou mobiles constituent une ressource précieuse au cycle 4 : elles proposent aux yeux des élèves des figurations du monde et facilitent ainsi leur perception des textes littéraires; elles sont également l'occasion de les confronter à des procédés sémantiques proches de ceux utilisés pour les textes et de développer des méthodes d'analyse spécifiques pour chacun d'entre eux; elles leur donnent accès à une culture complémentaire qui dialogue avec la culture littéraire et l'enrichit. (ibid., p. 236)

Certes, cette mise en dialogue de la littérature avec les arts iconographique et cinématographique est envisagée ici selon trois perspectives. Cependant, les images «facilitent [la] perception des textes littéraires », elles sont mises au service de la lecture de la littérature ; si elles permettent la confrontation de systèmes sémiotiques proches, la référence demeure malgré tout celui propre au langage littéraire; les images ressortissent à une culture " complémentaire ", qui « enrichit » la culture littéraire. Cette subordination des arts iconographique et cinématographique à la littérature s'explique par l'orientation disciplinaire retenue par l'institution dans les programmes, ceux-ci concernant ici l'enseignement du français au cycle 4 . Elle rejoint l'une des perspectives que les candidats au Capes peuvent privilégier. Lors de la première épreuve d'admission ${ }^{6}$ dite " mise en situation professionnelle » (désormais MSP), il leur est demandé de traiter un dossier composé d'un texte littéraire et d'un document iconographique (photographie, affiche de film, tableau, photogramme, frontispice, etc.), ce dernier « constituant une force d'appoint que les candidats doivent mettre en relation avec le texte étudié » (Rapport MSP, $2014^{7}$, p. 114). Et il est précisé que « l'adjonction d'un document iconographique conforte l'interdisciplinarité qui marque désormais l'enseignement secondaire, les programmes officiels accordant une place non négligeable à l'histoire de l'art et à l'étude de l'image, présentes à chaque niveau de l'enseignement au collège ${ }^{8} »$ (ibid., p. 115). L'analyse des rapports conduit à mettre au jour deux orientations, sachant que, dans les deux cas, ce document iconographique n'est à considérer que comme une aide complémentaire à l'étude du texte, car c'est bien celle-ci qui « doit absolument primer » (ibid.) : le candidat est invité à « montrer véritablement en quoi [l'image] peut enrichir la lecture du texte » ( ibid., p. 128). 
10 Dans le premier cas, l'image a une « valeur historique, informative, documentaire » (ibid.), elle est mise au service de la contextualisation et/ou de la caractérisation du texte : La valeur parfois historique, informative, documentaire de l'image peut rendre judicieux son examen en introduction, dans la phase de contextualisation du texte, soit qu'il s'agisse de présenter l'auteur si l'image permet d'évoquer les rapports étroits qu'un auteur entretenait avec les milieux artistiques de son temps [...].

[...] l'image peut entrer en écho avec le texte pour aider à en introduire la thématique ou pour en faire ressortir - par rapprochement ou par contraste - le registre, la tonalité (lyrique, satirique, burlesque...) ou l'esthétique générale, ce qui peut amener à une intéressante réflexion sur la transposition ou la formulation visuelle de procédés proprement textuels, ou inversement. (ibid.)

13 Le statut ancillaire de l'image se trouve souligné : l'accent est mis sur le texte, l'auteur et le contexte dans lequel ceux-ci prennent place. Si mise en dialogue il y a entre la littérature et les arts, celle-ci consiste à mener une « réflexion sur la transposition ou la formulation visuelle de procédés proprement textuels, ou inversement ». L'orientation prise ici (et, qui plus est, attendue des candidats, futurs professeurs) remet en question le sens à donner à cette " mise en dialogue » de la littérature avec les arts et peut conduire ensuite à certaines dérives dans les classes: l'analyse de l'image vise seulement à conforter celle du texte, les deux activités étant réduites à des relevés de « procédés ».

Dans le second cas, l'image offre une interprétation possible du texte qu'il revient au candidat d'interroger, et, on peut le supposer, de confronter à sa propre interprétation. Il est en effet précisé :

15 Au-delà du caractère illustratif de l'image, c'est donc sa fonction interprétative qu'il convient d'interroger. Faut-il rappeler que même une image illustrative, une mise en scène, une transposition filmique témoignent toujours d'une lecture singulière du texte par le peintre, le metteur en scène ou le réalisateur. Illustrer, c'est donc transposer, interpréter, faire ressortir - fidèlement ou au contraire, par un jeu de tensions et distorsions - une dimension de l'œuvre verbale qu'on estime en être une des clés : quel moment du récit l'artiste choisit-il de représenter, quel personnage choisit-il de mettre en avant, dans quelle posture, dans quel environnement ? (ibid., p. 129)

16 L'accent est mis sur la " lecture singulière » du texte que l'artiste-lecteur donne à voir. Les équivalences établies entre "illustrer ", " transposer ", " interpréter ", gagneraient certes à être éclairées de manière à lever chez les candidats toute confusion possible. Il leur est cependant demandé de considérer l'image, de quelque nature qu'elle soit, non comme la représentation iconographique du texte visant à le compléter, mais comme l'expression de la rencontre d'un texte et d'un lecteur singulier, comme l'actualisation' (Citton, 2007 ; Ahr, 2017) que ce dernier en propose dans un langage autre que verbal. Se trouve dès lors favorisée la mise en dialogue de la littérature et des arts visuels, ainsi que l'explique le rapporteur de cette même épreuve lors de la session 2015 : « [...] c'est bien pour favoriser ce dialogue entre deux langages différents, au lieu d'aboutir à une tautologie, que les deux éléments - texte et image - sont souvent empruntés à des époques différentes voire à des contextes radicalement surprenants dans leur mise en parallèle » (Rapport MSP, 2015, p. 134).

17 Il s'agit bien, dans le cas présent, d'interroger cet échange, ce « dialogue entre deux langages différents» et non de subordonner l'un à l'autre. Cet infléchissement est confirmé lors de la session suivante : «Il s'agit de faire dialoguer le texte et l'image, de 
mettre en écho deux systèmes de signes, dont les résonances réciproques peuvent stimuler l'interprétation. [...] On le voit, l'explication de texte et l'analyse de l'image s'infusent et s'enrichissent réciproquement » (Rapport MSP, 2016, p. 129-130).

18 La seconde épreuve, appelée " Analyse d'une situation professionnelle » (désormais ASP), repose sur l'étude d'un dossier composé de textes et de documents complémentaires, notamment iconographiques dans le cadre des options Langue et littérature françaises (tableaux, gravures, photographies, photogrammes, planches de bande dessinée, dessins de presse, etc.), Théâtre (captations de mises en scène) et Cinéma (séquences filmiques). Cette seconde épreuve orale ${ }^{10}$ tend à "saisir la Littérature dans le cadre du dialogue qu'elle suscite entre les auteurs, les œuvres et avec d'autres formes artistiques » (Rapport ASP Littérature et langue françaises, 2014, p. 180). Quelle que soit l'option, le candidat doit «mettre en relation entre eux dans un dialogue fécond et convaincant " (Rapport ASP Cinéma, 2014, p. 266) les différents documents qui composent le corpus et dont la diversité «traduit la richesse de l'ouverture culturelle dans les multiples possibilités d'éclairage du corpus qu'elle induit, à la croisée des arts » (Rapport ASP Littérature et langue françaises, 2015, p. 157).

Qu'il s'agisse des corpus soumis à l'analyse des candidats ou bien de ceux élaborés par les enseignants à destination de leurs élèves, ceux-ci ouvrent l'enseignement de la littérature aux « autres formes d'expression artistique (particulièrement des œuvres picturales et cinématographiques) », comme le précisent les programmes de français du cycle 4 (MENESR, 2015c, p. 247). Cette restriction s'explique certes par des raisons d'ordre matériel (au concours comme dans les classes), mais elle limite « l'ouverture culturelle et artistique » (Rapport ASP Littérature et langue françaises, 2015, p. 148) que les discours institutionnels s'efforcent de promouvoir. La mise en place du PÉAC à tous les niveaux de la scolarité et celle des EPI au cycle 4, qui reposent tous deux sur le principe de l'interdisciplinarité, tendront peut-être à pallier ce déséquilibre qui affecte les «autres formes d'expression artistique ». Le Guide pour la mise en cuvre du parcours d'éducation artistique et culturelle (MEN, 2013b, p. 8) rappelle en effet un des principes majeurs du PÉAC :

20 Au-delà de la progressivité du parcours, les enseignants ont aussi la responsabilité, collectivement et individuellement, de veiller à la diversité des grands domaines artistiques et culturels abordés par les élèves tout au long de leur scolarité - différentes formes du spectacle vivant, arts visuels, arts du son, arts de l'espace, arts appliqués, arts du langage. Par leurs choix pédagogiques (œuvres étudiées, lieux explorés, intervenants, professionnels des arts et de la culture rencontrés etc.), ils s'assurent en outre que les élèves explorent ces domaines dans leurs manifestations patrimoniales et contemporaines, pour permettre un dialogue fécond entre l'art d'hier et l'art d'aujourd'hui.

Effectivement, la diversité concerne tout aussi bien les formes artistiques que les « formes patrimoniales et contemporaines, populaires et savantes » des œuvres, considérées dans leurs "dimensions nationales et internationales", troisième des dix principes autour desquels s'organise la Charte pour l'éducation artistique et culturelle signée par quatre ministères (MENESR et al., 2016). Les nouveaux programmes de français du cycle 4 préconisent aussi cette diversité :

Chaque année, le professeur aborde les questionnements au programme en mobilisant les ressources de: la littérature patrimoniale (en s'efforçant de puiser dans toutes les époques, du Moyen Âge au $\mathrm{XX}^{\mathrm{e}}$ siècle); la littérature contemporaine; les littératures 
antiques et étrangères; les littératures francophones; la littérature de jeunesse; les textes non littéraires de natures et de fonctions variées (écrits sociaux, documentaires). Il exploite aussi des œuvres issues de domaines artistiques diversifiés. Il s'agit notamment d'établir constamment des ponts entre le passé, le présent et les questions du monde de demain, en dépassant les frontières artificielles, dans une perspective culturelle ouverte et riche. (MENESR, 2015c, p. 237-238)

Les "programmes de français et littérature » actuels du lycée général et technologique favorisent également l'ouverture de l'enseignement des lettres aux autres arts dans toute leur diversité. Il est par exemple recommandé aux professeurs, dans le cadre de l'objet d'étude "Le personnage de roman, du XVII ${ }^{e}$ siècle à nos jours ${ }^{11}$ » en classe de première, d'opérer « un choix de textes et de documents permettant de mettre en évidence les relations existant entre la représentation des personnages et des milieux romanesques et celle qu'en donnent les autres arts - la peinture, la gravure, la sculpture, l'opéra par exemple - à la même époque» (MEN, 2010). Cependant, cette dernière précision réduit le choix des possibles et entre en contradiction avec le libellé même de l'objet d'étude. Par ailleurs, on peut lire dans le Préambule de ces programmes:

Les programmes de français et littérature en classes de seconde et première répondent à des objectifs qui s'inscrivent dans les finalités générales de l'enseignement des lettres au lycée : l'acquisition d'une culture, la formation personnelle et la formation du citoyen. Ces finalités sont atteintes grâce à une progression méthodique qui prend appui principalement sur la lecture et l'étude de textes majeurs de notre patrimoine.

Quels sont ces " textes majeurs de notre patrimoine », sachant que si les rapprochements avec les langues et cultures de l'Antiquité sont fortement conseillés, la lecture et l'étude de la littérature étrangère, en particulier européenne, n'est prescrite que pour la classe de première littéraire? Il est précisé sur ce point: «La prise en compte d'un espace culturel européen permet de familiariser les élèves avec quelques textes de référence partagés et de créer des échos entre ces deux heures d'enseignement spécifique et les deux heures de "littérature étrangère en langue étrangère" ".

Et même si les corpus soumis à l'étude des candidats au Capes semblent s'ouvrir à la littérature contemporaine - J. Echenoz et M. Ndiaye sont cités dans la présentation du rapport de 2014 (Blanckeman, 2014, p. 6) - la perspective d'ouverture culturelle et artistique, qui sous-tend les discours institutionnels examinés dans le cadre de cet article, semble susciter des tensions internes que les enseignants doivent apprendre à gérer, tout comme les candidats au concours de recrutement: mise en dialogue des œuvres littéraires et des « autres formes d'expression artistiques » ou mise en dialogue des œuvres littéraires et " particulièrement des œuvres picturales et cinématographiques » (MENESR, 2015c, p. 247) ? Cette mise en dialogue concerne-t-elle les œuvres artistiques participant de la culture savante et/ou populaire ? Comment expliquer la contradiction relevée au sein d'un même rapport (ASP Théâtre, 2016) au sujet de l'enseignement du théâtre? On peut lire page 230 :

27 Le jury précise qu'il est souhaitable d'enrichir l'approche trop académiquement scolaire du texte théâtral. Bien entendu, l'épreuve ne disqualifie pas l'approche littéraire du texte théâtral, mais elle affiche l'ambition d'en renouveler l'approche scientifique et pédagogique en tenant compte des apports majeurs des recherches universitaires en études théâtrales depuis leur fondation dans les années 1950-1960 et en se situant résolument à l'articulation du texte et de sa représentation. 

quant à la nécessité de favoriser en classe la rencontre sensible avec l'œuvre artistique, quelle qu'en soit la forme, ainsi que «la parole sur l'œuvre» (Chabanne, 2012, p. 16-30), celle-ci devant conjuguer "dimension sensible et dimension rationnelle», comme le soulignent les programmes de 2015. artistique, par la fréquentation des œuvres et par l'expression de ses émotions et de ses gouts. L'histoire des arts, qui associe la rencontre des œuvres et l'analyse de leur langage, contribue à former un lien particulier entre dimension sensible et dimension rationnelle. En français, on s'attache à permettre la réception sensible des œuvres littéraires en développant son expression, la formulation de ses opinions, dans des échanges oraux ou en en recueillant les traces écrites ${ }^{12}$ dans des carnets de lecture ${ }^{13}$. (MENESR, 2015c, p. 95)

L'un des champs dans lesquels s'inscrivent les objectifs généraux de l'enseignement de l'histoire des arts, auquel le professeur de français contribue activement, est «d'ordre esthétique, relevant d'une éducation de la sensibilité » (MENESR, 2015c, p. 148 pour le cycle 3, p. 284 pour le cycle 4). Il s'agit, entre autres, de développer chez l'élève « des attitudes qui permettent d'ouvrir sa sensibilité à l'œuvre d'art", mais aussi de "développer des liens entre rationalité et émotion » (ibid. : p. 149, p. 286). Le domaine 5, «Les représentations du monde et l'activité humaine», du socle commun de 
connaissances, de compétences et de culture, vise notamment au "développement cognitif et sensible » de l'élève :

Il [l'élève] exprime à l'écrit et à l'oral ce qu'il ressent face à une œuvre littéraire ou artistique; il étaye ses analyses et les jugements qu'il porte sur l'œuvre; il formule des hypothèses sur ses significations et en propose une interprétation en s'appuyant notamment sur ses aspects formels et esthétiques. Il justifie ses intentions et ses choix expressifs, en s'appuyant sur quelques notions d'analyse des œuvres. [...] Il développe son jugement, son goût, sa sensibilité, ses émotions esthétiques. (MENESR, 2015a, p. 7) Ces orientations institutionnelles rejoignent celles qui sous-tendent, au moins partiellement, les programmes du lycée, l'une des six finalités de l'enseignement des lettres étant « le développement d'une conscience esthétique permettant d'apprécier les œuvres, d'analyser l'émotion qu'elles procurent et d'en rendre compte à l'écrit comme à l'oral » (MEN, 2010). C'est d'ailleurs cette finalité que le rapporteur de l'épreuve ASP Littérature et langue françaises de la session 2014 rappelle aux futurs candidats en ces termes :

La présence significative de reproductions picturales, mais aussi d'autres types d'iconographies, n'est pas un prétexte à une séance d'« Histoire des Arts ", comme le jury l'a trop souvent entendu. Il s'agit au contraire de donner l'occasion au candidat de montrer qu'il est capable d'éveiller et d'enrichir la conscience esthétique de ses futurs élèves. (p. 180)

Il est difficile de penser que, pour les différents acteurs de l'institution, émotion et appréciation doivent nécessairement être consensuelles! Et pourtant, il y a lieu de s'interroger sur la nature de la rencontre avec l'œuvre et celle de la parole qui en témoigne attendues par les jurys dont la mission est de recruter les professeurs de lettres.

Force est d'admettre tout d'abord que, lors du concours, la rencontre avec l'œuvre n'est pas une expérience authentique et cela au moins pour deux raisons. L'œuvre présentée au candidat est un « avatar ", tel que J.-C. Chabanne le définit (2012, p. 10), c'est-à-dire une reproduction de l'œuvre originelle, comme le plus souvent dans les classes. De plus, cette rencontre se réalise dans des conditions fort particulières, celles d'un concours de recrutement, et elle répond à un enjeu précis : montrer au jury l'étendue de sa culture artistique et la maitrise des compétences requises pour enseigner les «lettres», car il s'agit bien d'un Certificat d'aptitude au professorat de l'enseignement du second degré (Capes) de lettres. Peut-on dès lors parler d'une rencontre avec les œuvres, que cette rencontre soit définie comme une simple «mise en contact» ou comme une véritable "expérience esthétique» (Schaeffer, 2015), distinction que J.-C.Chabanne établit également (2012, p.16)? Par ailleurs, la parole sur l'œuvre est nécessairement conditionnée par les attendus du concours, qui diffèrent selon les rapporteurs. De fait, ces écarts reflètent les tensions qui marquent, encore aujourd'hui, l'enseignement scolaire de la littérature (Ahr, 2015). Celui-ci doit-il en effet privilégier une approche centrée sur les œuvres littéraires, voire artistiques, et une lecture experte de celles-ci et/ou une approche centrée sur la réception sensible de ces œuvres par les lecteurs/spectateurs que sont les élèves? Les écarts constatés d'une commission à l'autre, d'une année à l'autre, reposent sur des conceptions différentes de la littérature et de l'art et surtout des finalités de leur transmission. 

de l'analyse filmique, qui réduirait l'interprétation des images à un jeu d'identification de figures de style, sans autre fin que lui-même. La mobilisation des outils d'analyse filmique (raccords, échelles des plans, mouvements de caméra...) n'a d'intérêt (pour les candidats et pour les élèves dont ils auront la charge) que si elle est reliée à une réflexion sur les effets produits, l'émotion qu'elle suscite et les significations qu'elle dégage, pour aboutir à une hypothèse interprétative d'ensemble, qui mette en valeur des choix de mise en scène au service d'une vision du monde. (p. 243-244)

Certaines commissions adoptent donc une position en adéquation avec les prescriptions institutionnelles récentes, puisqu'elles semblent favoriser la rencontre avec l'œuvre comme expérience esthétique et la parole sur l'œuvre comme mise en mots de cette expérience, si l'on admet que celle-ci se définit comme une "expérience attentionnelle exploitant nos ressources cognitives et émotives » (Schaeffer, 2015, p. 45). Il est ainsi rappelé aux candidats que « fréquenter quotidiennement les textes, en donner le goût aux élèves, leur en donner l'accès par une lecture sensible et raisonnée est au cœur du métier de professeur de français» (Rapport MSP, 2014, p.115). Il leur est conseillé de "se constituer une connaissance sensible» (Rapport ASP Théâtre, 2015, p. 239) des œuvres artistiques, quelle que soit leur forme - et pas seulement dans le cadre des arts du 
spectacle vivant. Effectivement, comme le démontre J.-M. Schaeffer (2015, p.141-142) dans son dernier essai L'expérience esthétique :

[...] la cognition et les émotions forment deux systèmes structurellement corrélés. Les situations dans lesquelles l'un des systèmes est activé sans que l'autre le soit sont extrêmement rares. S'il existe quelques situations, tels la neutralité axiologique de l'enquête scientifique, l'intellectualisation ou le détachement, dans lesquelles il est possible de dissocier l'évaluation cognitive de l'évaluation émotive, il n'existe en revanche pas de processus émotionnel dépourvu de dimension cognitive: [...] toute émotion est le produit direct ou indirect (dans le cas des réactions évaluantes innées) d'une évaluation cognitive, même si elle n'est pas toujours consciente, ni toujours fiable ${ }^{14}$.

[...] Des travaux de psychologie expérimentale menés dans les années 1960, notamment par Berlyne, ont montré que lorsqu'une activité de mémorisation est liée à une évaluation émotive l'efficacité de la mémorisation augmente : des contenus associés à une émotion sont moins vite oubliés que des contenus neutres.

"Se constituer une connaissance sensible» des œuvres artistiques pour certains rapporteurs, "développer une conscience esthétique » chez les collégiens et les lycéens ne passent pas seulement par l'assimilation de savoirs. Cela passe aussi par la rencontre sensible avec les œuvres, au sens de « faire l'expérience esthétique des œuvres », qu'elles soient littéraires ou plastiques voire musicales, ainsi que par le partage entre pairs de ces évènements esthétiques, la confrontation favorisant un retour réflexif sur la réception première des œuvres, comme le montrent la sociologie de l'art (Le Quéau, 2007 ; Fleury, 2007) ainsi que certains travaux menés dans le champ de la didactique de la littérature (Ahr, 2013 ; Gabathuler, 2016 ; Shawky-Milcent, 2016).

\section{En guise de conclusion}

L'analyse des rapports des trois dernières sessions du Capes met au jour certaines contradictions, qui reflètent des conceptions différentes - ou, tout du moins, non stabilisées - de l'enseignement scolaire de la littérature, notamment en lien avec les autres langages artistiques. Ces contradictions sont à la fois internes, puisqu'on relève des orientations divergentes au sein des diverses commissions ou bien d'une année sur l'autre, et externes, si l'on met certaines de ces orientations en écho avec celles privilégiées par le ministère de l'Éducation nationale dans ses textes de cadrage récents. On relève également dans ces derniers quelques ambiguïtés, qu'il conviendrait de lever, et certaines options qui peuvent limiter le processus d'ouverture culturelle et artistique vers lequel doit tendre désormais l'enseignement de la littérature dans le secondaire. On gagnerait donc à harmoniser les discours institutionnels analysés dans le cadre de cet article, même si l'on sait qu'ils sont tenus dans des sphères, si ce n'est opposées, du moins distinctes. La mise en dialogue de la littérature avec les arts peut contribuer à la transformation des pratiques enseignantes, en accordant une plus grande place à la réception «sensible et raisonnée " des œuvres, indépendamment de leur forme artistique. Pour atteindre cet objectif, un certain nombre de notions et de concepts, très présents dans les rapports de jurys et dans les prescriptions institutionnelles examinés, sont non seulement à préciser et à harmoniser, mais également à définir en termes d'activités de classe. Par ailleurs, sont à mesurer les effets induits par ces activités nouvelles, impulsées par les discours institutionnels, sur la formation des élèves en tant que sujets de culture. Il revient aux chercheurs, aux formateurs et aux enseignants de 
s'emparer de ces questions d'une profonde actualité institutionnelle mais aussi sociale. Comme le rappelle en effet J.-C. Léon (2017) en ouverture du numéro 535 des Cahiers pédagogiques, "la culture est, parmi ses multiples acceptions, ce qui fait lien, ce qui permet aux humains de vivre ensemble ».

\section{BIBLIOGRAPHIE}

AHR, S. (éd.) (2013). Vers un enseignement de la lecture littéraire au lycée. Expérimentations et réflexions. Grenoble : CNDP/CRDP Grenoble.

AHR, S. (2015). Enseigner la littérature aujourd'hui. « Disputes » françaises. Paris : H. Champion.

AHR, S. (2017). «Actualisation / (re)contextualisation des textes patrimoniaux : d'un questionnement épistémologique à des questions d'ordre didactique », Recherche et Travaux 91. En ligne : https://recherchestravaux.revues.org/954 - quotation.

AHR, S. (sous presses). «Littérature et arts : quelle mise en dialogue au Capes de lettres modernes? In : Diptyque. Namur : Presses universitaires de Namur.

BLANCKEMAN, B. (présenté par le président du jury) $(2014,2015)$. Rapport de jury du concours externe du Capes et Cafep de lettres. En ligne : http://media.devenirenseignant.gouv.fr/file/ capes_ext/78/5/lettres_350785.pdf; http://media.de venirenseignant.gouv.fr/file/ capes_ext/70/5/lettres_473705.pdf.

CHABANNE, J.-C. (2012). « Introduction ». In : Chabanne, J.-C., Parayre, M. \& Villagordo, É. (éds.), La Rencontre avec l'œuvre. Éprouver, pratiquer, enseigner les arts et la culture. Paris : L'Harmattan, p. 7-47. CITTON, Y. (2007). Lire, interpréter, actualiser. Pourquoi les études littéraires ? Paris : Éd. Amsterdam.

FLEURY, L. (2007). «L'art, l'émotion et les valeurs : Contribution d'une sociologie des émotions à la sociologie de l'art et de la culture ». In : Le Quéau, P. (éd.), 20 ans de sociologie de l'art, tome 2. Paris : L'Harmattan, p. 149-161.

GABATHULER, C. (2016). Apprécier la littérature. La relation esthétique dans l'enseignement de la lecture de textes littéraires. Rennes : Presses universitaires de Rennes.

LAUDET, P. (présenté par le président du jury) (2016). Rapport de jury du concours externe du Capes et Cafep de lettres. En ligne : http://media.devenirenseignant.gouv.fr/file/ externe/89/5/rj-2016capes-externe-lettres-admission_633895.pdf.

LE QUÉAU, P. (2007). « La formation des émotions ». In : Le Quéau, P. (éd.), 20 ans de sociologie de l'art, tome 2. Paris : L'Harmattan, p. 163-178.

LÉON, J.-C. (coord.) (2017). « Arts et culture : quels parcours ? ». Cahiers pédagogiques 535.

MINISTÈRE DE L'ÉDUCATION NATIONALE (2008). Bulletin officiel no 32 du 28 août 2008. Organisation de l'enseignement de l'histoire des arts à l'école primaire, au collège et au lycée. En ligne : http:// cache.media.education.gouv.fr/file/32/09/0/encart_33090.pdf. 
MINISTÈRE DE L'ÉDUCATION NATIONALE (2009). Bulletin officiel spécial nº 2 du 19 février 2009. Programme d'enseignement de français pour les classes préparatoires au baccalauréat professionnel En ligne : http://media.education.gouv.fr/file/special_2/ 24/5/francais_44245.pdf. MINISTÈRE DE L'ÉDUCATION NATIONALE (2010). Bulletin officiel spécial no 9 du 30 septembre 2010. Programme de l'enseignement commun de français en classe de seconde générale et technologique et en classe de première des séries générales et programme de l'enseignement de littérature en classe de première littéraire. En ligne : http://www.education.gouv.fr/cid53318/ mene1019760a.html.

MINISTÈRE DE L'ÉDUCATION NATIONALE (2013a). Circulaire interministérielle 2013-073 du 3 mai 2013, Bulletin officiel $\mathrm{n}^{\circ} 19$ du 9 mai 2013. Actions éducatives. Parcours d'éducation artistique et culturelle. En ligne : http://www.education.gouv.fr/pid25535/bulletin_officiel. html? cid_bo=71673.

MINISTÈRE DE L'ÉDUCATION NATIONALE (2013b). Guide pour la mise en œuvre du parcours d'éducation artistique et culturelle. En ligne : http://cache.media.eduscol.education.fr/file/ Domaines_artistiques/49/9/2013_EAC_guide_bdef_287499.pdf.

MINISTÈRE DE L'ÉDUCATION NATIONALE, DE L'ENSEIGNEMENT SUPÉRIEUR ET DE LA RECHERCHE (2015a). Décret $\mathrm{n}^{\circ}$ 2015-372 du 31/03/2015. Journal officiel du 02/04/2015. Socle commun de connaissances, de compétences et de culture. En ligne : http://www.education.gouv.fr/pid25535/ bulletin_officiel.html?cid_bo=87834.

MINISTÈRE DE L'ÉDUCATION NATIONALE, DE L'ENSEIGNEMENT SUPÉRIEUR ET DE LA RECHERCHE (2015b). Arrêté du 01/07/2015. Journal officiel du 07/07/2015. Actions éducatives. Parcours d'éducation artistique et culturelle. En ligne : http://www.education.gouv.fr/pid25535/bulletin_officiel.html? cid_bo=91164.

MINISTÈRE DE L'ÉDUCATION NATIONALE, DE L'ENSEIGNEMENT SUPÉRIEUR ET DE LA RECHERCHE (2015c). Bulletin officiel spécial $\mathrm{n}^{\circ} 11$ du 26 novembre 2015. Programmes d'enseignement du cycle des apprentissages fondamentaux (cycle 2), du cycle de consolidation (cycle 3) et du cycle des approfondissements (cycle 4). En ligne : http://cache.media.education.gouv.fr/file/ MEN_SPE_11/67/3/2015_programmes_cycles234_4_12_ok_508673.pdf.

MINISTÈRE DE L'ÉDUCATION NATIONALE, DE L'ENSEIGNEMENT SUPÉRIEUR ET DE LA RECHERCHE, MINISTÈRE DE LA CULTURE ET DE LA COMMUNICATION, MINISTÈRE DE LA VILLE, DE LA JEUNESSE ET DES SPORTS, MINISTÈRE DE L'AGRICULTURE, DE L'AGROALIMENTAIRE ET DE LA FORÊT (2016). Charte pour l'éducation artistique et culturelle. En ligne : http://eduscol.education.fr/cid105396/charte-pour-education-artistiqueculturelle.html.

ROHAN-CSERMAK (de), H. (2010). « La mise en œuvre de l'enseignement de l'histoire des arts en France ». En ligne : http://eduscol.education.fr/histoire-des-arts/se-former/regards-surlhistoire-des-arts/la-mise-en-oeuvre-de-lenseignement-de-lhistoire-des-arts-en-france.html. SCHAEFFER, J.-M. (2015). L'expérience esthétique. Paris : Gallimard.

SHAWKY-MILCENT, B. (2016). La lecture, ça ne sert à rien! Usages de la littérature au lycée et partout ailleurs. Paris : Presses universitaires de France.

\section{NOTES}

1. En France, pour enseigner dans un collège ou un lycée général et technologique publics, sont requis, hormis certaines conditions particulières, le Certificat d'aptitude au professorat de 
l'enseignement du second degré (Capes), concours de la fonction publique qui peut être préparé au sein d'une École supérieure du professorat et de l'éducation (ESPÉ), ainsi qu'un master seconde année et la validation d'une année de stage. Voir à ce sujet : http:// www.devenirenseignant.gouv.fr/pid33985/enseigner-college-lycee-general-capes.html.

2. Pour cette seconde épreuve du Capes de lettres modernes, les candidats ont le choix entre plusieurs options : latin, littérature et langue françaises, français langue étrangère et français langue seconde, théâtre, cinéma.

3. La présente contribution reprend et approfondit certains des éléments développés dans un autre article, rédigé antérieurement à la publication du rapport de jury de la session 2016, celuici faisant explicitement allusion aux programmes des cycles 3 et 4 entrés en application à la rentrée 2016: S. Ahr (sous presse), «Littérature et arts : quelle mise en dialogue au Capes de lettres modernes?».

4. Le choix est fait, dans la suite de l'article, de ne pas appuyer notre propos sur les programmes du lycée professionnel dans la mesure où une partie de notre corpus est constitué des rapports de jurys du concours de recrutement des professeurs de collège et de lycée. Ces programmes de 2009 ainsi que les ressources qui les accompagnent annoncent cependant les orientations prises par l'institution au cours de la décennie actuelle.

5. Voir par exemple l'article du Monde du 29/06/2012 : http://www.lemonde.fr/ecole-primaireet-secondaire/article/2012/06/29/1-epreuve-d-histoire-des-arts-s-installe-au-brevet-descolleges-dans-un-certain-desordre_1726981_1473688.html.

6. Les deux premières épreuves écrites d'admissibilité (Composition française et Étude grammaticale de textes de langue française) évaluent respectivement la culture littéraire et artistique et les compétences en grammaire scolaire des candidats. La première épreuve orale d'admission (Épreuve de mise en situation professionnelle) consiste en une explication de texte assortie d'une question de grammaire scolaire ; la seconde épreuve orale d'admission (Analyse d'une situation professionnelle) vérifie la capacité des candidats à analyser les documents qui leur sont proposés et à les inscrire dans une démarche d'enseignement et d'apprentissage en relation avec les programmes. Le descriptif des épreuves est consultable sur le site du ministère : http://www. devenirenseignant.gouv.fr/cid98573/les-epreuves-du-capes-externe-et-du-cafepcapes-section-lettres.html.

7. C'est le rapporteur qui souligne. Le choix est fait, dans cet article, de ne pas citer les rapporteurs. Le référencement ne porte donc que sur la nature de l'épreuve d'admission et l'année de la session.

8. Cette précision est étrange dans la mesure où les classes de lycée sont tout autant concernées par cette "interdisciplinarité ». On notera également qu'il s'agit d'accorder " une place non négligeable à l'histoire de l'art » et non des arts.

9. L'emploi de ce terme serait préférable à ceux de «transposition» et d'«illustration». Il conviendrait de lever les flous terminologiques et, de fait, conceptuels présents dans ces rapports comme dans certaines instructions officielles.

10. On ne s'intéresse ici qu'à ces trois options.

11. Nous soulignons ici, comme dans les citations qui suivent.

12. «ou les traces iconographiques ou même encore sonores", conviendrait-il d'ajouter.

13. " ou en favorisant la pratique même de l'écriture voire de toute autre expression artistique ", conviendrait-il également d'ajouter.

14. C'est l'auteur qui souligne. 


\section{RÉSUMÉS}

Nombreux sont les discours institutionnels, publiés ces toutes dernières années, qui tendent à promouvoir la mise en dialogue de l'enseignement de la littérature avec les arts. Ces discours concernent tout aussi bien la classe que la formation universitaire préparant au concours de recrutement des enseignants de français. Après une rapide présentation du corpus constitué, l'analyse comparée des rapports de jury des deux épreuves orales du Capes externe de lettres modernes des trois dernières sessions (2014-2016) et des divers textes officiels cadrant aujourd'hui l'enseignement de la littérature dans le secondaire conduit à mettre au jour les convergences et les divergences perceptibles dans ces discours institutionnels, qui, d'une façon ou d'une autre, déterminent les pratiques de classe en matière d'enseignement de la littérature en lien avec les autres œuvres artistiques.

Numerous institutional discourses have been issued these last years which tend to promote a dialogue between literature teaching and arts. These discourses concern schooling as well as the training of student teachers in French for recruitment exams at university. After a short presentation of the submitted corpus the analysis compares on the one hand examiners'reports of both oral exams of the Capes externe de lettres modernes (national recruitment exam of French teachers) from the three previous years (2014-2106) and on the other hand varied extracts from the national curriculum for French literature at secondary school. This compared analysis leads us to highlight the points of convergence and divergence which are perceptible in these institutional discourses which determine in a way or another the teaching practice in literature along with other art forms.

\section{INDEX}

Mots-clés : littérature et arts, enseignement secondaire, formation universitaire, discours institutionnels

Keywords : literature and arts, secondary school, university education, institutional discourses

\section{AUTEUR}

\section{SYLVIANE AHR}

Université Toulouse Jean-Jaurès, LLA/CRÉATIS, ESPÉ Midi-Pyrénées, F-31000, France 